\title{
Analysis of the thermal environment inside the furnace of a dynamic mechanical analyser
}

\author{
N.M. Alves ${ }^{\text {a }}$, J.F. Mano ${ }^{\text {a,* }}$, J.L. Gómez Ribelles ${ }^{\text {b }}$ \\ a Polymer Engineering Department, University of Minho, Campus of Azurém, 4800-058 Guimarães, Portugal \\ ${ }^{\mathrm{b}}$ Center for Biomaterials and Department of Applied Thermodynamics, Universidad Politécnica de Valencia, P.O. Box 22012, E- \\ 46071 Valencia, Spain
}

Received 14 June 2002; accepted 15 September 2002

\begin{abstract}
In this work, the thermal environment inside the furnace of a dynamic mechanical analyser was investigated by putting a standard sample in distinct positions inside the furnace. Penetration experiments were carried out in order to compare the measured melting temperature of the sample with the theoretical melting temperature. The thermal gradients were investigated for three distinct modes: compression, three-point bending and extension. In the compression mode a small variation of the measured melting point was found as a function of the radial distance. However, for both extension and three-point bending modes quite important variations were measured along the relevant directions. In the former case the bottom clamp was found to be warmer than the upper one and in the latter mode higher temperatures are found in the extremities of the samples (closer to the furnace wall). Other factors, such as the influence of the heating rate and the purge gas flow rate, were also investigated. In the particular case of the extension mode, it was found that the measured melting temperature decreased with increasing scanning rate. A simple model was used in order to investigate the influence of the temperature gradients on DMA measurements. For temperature gradients along the sample length below $10^{\circ} \mathrm{C}$ the differences in the viscoelastic parameters $(\tan \delta, D *$ and $E *$ ) from the homogeneous case are small when compared with typical experimental errors.
\end{abstract}

(c) 2002 Elsevier Science Ltd. All rights reserved.

Keywords: DMA; TMA; Thermal gradients; Calibration; Temperature correction; Polymeric samples

\section{Introduction}

The absolute accuracy and repeatability of the results obtained in a dynamic mechanical analyser (DMA) depends on many factors, for example the material properties, the sample shape, the sample alignment, the clamping arrangement, the oscillation amplitude, the system used to cut the sample or the presence of vibrations close to the equipment [1-4]. A Taguchi experimental

\footnotetext{
* Corresponding author. Tel.: +351-253-510245; fax: $+351-$ 235-510249.

E-mail address: jmano@dep.uminho.pt (J.F. Mano).
}

design has been proposed in [4] to improve the measurement process of a DMA.

Among these factors a major aspect regarding the accuracy of the experiments performed in a DMA is the precise knowledge of the sample temperature as the physical properties are monitored. It must be pointed out that it is not possible to attribute a single temperature to the sample because of its own resistance, especially if it is a bad heat conductor, so in practice there are distinct temperatures at distinct points of the sample and the designation true or real sample temperature that appears in literature is in fact an average sample temperature. In DMA the temperature sensor is close to but separated by a gas gap from the sample. Differences between the sample temperature and the temperature read by the 
sensor are due to different factors that include the set of thermal resistances (sample, accessories, purge gas), the heat fluxes inside the furnace and the accuracy of the sensor reading.

The melting of high purity standards, usually metals, has been widely used for the temperature calibration of DMA and thermomechanical analysers (TMA) [5]. In this case the measured onset is compared with the corresponding melting points of the standards. The experiments are conducted in the penetration or compression modes and two standards (two-point calibration) with melting in the temperature range of interest should be preferably used [6,7]. An abbreviated procedure is the one-point calibration, where only one standard, also with a melting point near the temperature of the transition under study, is used [6].

Other methods of calibration for these equipments have been proposed. For example, Kociba [8] proposed a method of calibration for TMA using Curie temperature reference standards. The author found that the use of these standards provides an accurate and precise method of temperature calibration. Lotti and Canevarolo [9] present a temperature calibration procedure for a DMA using pure, non-metallic, substances with known melting points. The standards are placed in tubes of PP (for subambient temperatures) or PTFE (for higher temperatures) long enough to be fixed in the sample holder frame. The mode of deformation used is 3-point bending or dual cantilever. The melting point of the standard is read at the maximum of the tan $\delta$ curve and compared with the expected theoretical value. With the measured melting temperatures of several standards covering a large temperature range they obtain a calibration curve for the equipment. A new method for calibrating TMA in the extension mode was proposed and used for fibres [10] with a system that permits performing both TMA and differential thermal analysis (DTA). An indium pellet was cut into two pieces, flattened and pressed onto the centre of a PET fibre with the fibre in between. The temperature sensor was placed towards the centre of the fibre, $3 \mathrm{~mm}$ in distance and the run was performed with a preheated fibre. The difference between the onset measured by DTA and the theoretical melting point of indium is the needed temperature correction.

In most of the works reporting aspects of temperature calibration of DMA and TMA equipment, the analysis is carried out at a single position in the furnace. However, it is expected to find different temperatures at different points, even at isothermal conditions, because of the existence of complex heat fluxes resulting from the existence of hot and cold sources and a flux of a purge gas. The reliability of DMA or TMA should also then include information about the gradients along the sample geometry that may affect the overall measured response of the material. In this work the thermal lag effect is investigated by putting a standard sample in distinct pos- itions inside the furnace in accordance with the position of the samples that are currently analysed in the equipment. Future studies should include the investigation of the melting of standards along the sample's thickness in order to access to the thermal gradients inside the materials.

The dimensions of the sample used in DMA and TMA experiments are significant with respect to the dimensions of the furnace. Therefore, one should expect the existence of temperature gradients throughout the surface of a sample.

Moreover, DMA and TMA usually have different modes of operation (bending, extension, torsion, compression) in order to analyse samples with different geometries and properties. Therefore, the change of the accessories associated with each mode and the corresponding different arrangement of the sample in the furnace will create a completely distinct environment felt by the sample, even if the experimental conditions are the same. In this work the different modes of operating the DMA will also be taken into account in the description of the thermal gradients. The influences of the heating rate and the gas flux in the magnitude of the thermal gradients are also analysed.

Note that as the materials usually investigated by DMA or TMA have low thermal conductivity, a thermal gradient is expected from the surface to the centre of the piece, mainly in non-isothermal conditions. The penetration experiments performed in this work probe essentially the thermal environment throughout the surface of a sample, because metallic standards of small dimensions are used.

\section{Experimental}

\subsection{Equipment}

In this work the experiments were carried out in a DMA7e Perkin Elmer dynamic mechanical analyser with controlled cooling accessory. Unless otherwise stated, the experiments were performed using a high purity Helium as purge gas with a flow rate of $28 \mathrm{~cm}^{3} \mathrm{~min}^{-1}$ to improve heat transfer throughout the sample surroundings during the experiments. All the runs were conducted without temperature calibration, except for the initial temperature calibration of the thermocouple performed by Perkin Elmer manufacturers. The mechanical calibrations described in the equipment's manual were carried out prior to the experiments.

\subsection{Static experiments}

The TMA mode of this analyser was selected and a static force of $10 \mathrm{mN}$ and an indium sample (supplied by Goodfellow, catalogue number IN007921, 
purity $=99.99999 \%$, theoretical melting point $=156.60^{\circ} \mathrm{C}$ ) with $\sim 20 \mathrm{mg}$ of mass and with a cubic geometry were used. The thermal gradients were investigated for three distinct accessories: compression, three-point bending and extension.

\subsection{1. (i) Compression}

The $20 \mathrm{~mm}$ parallel plate accessory was used (see Fig. 1). Several experiments with and without a circular film of polyimide (PI) between the plates (with the same diameter as the plates) were performed. The studies with PI pretend to analyse the temperature of the surface of typical polymeric materials with low thermal conductivity. The effect of the distance from the centre of the plate in the measured melting point $\left(T_{\mathrm{m}}\right)$ of an indium sample and the effect of the heating rate $\beta=\mathrm{d} T / \mathrm{d} t$ $\left(1 \leq \beta \leq 8^{\circ} \mathrm{C} \min ^{-1}\right.$ in $T_{\mathrm{m}}$ for two positions (the centre of the plate and $9 \mathrm{~mm}$ away from the centre) were studied.

\subsection{2. (ii) Three-point bending}

The effect of $\beta\left(1 \leq \beta \leq 8^{\circ} \mathrm{C} \mathrm{min}{ }^{-1}\right.$ in $T_{\mathrm{m}}$ was investigated using the arrangement described in Fig. 2(a) with a bending platform of $15 \mathrm{~mm}$. Two distinct probe tips (3 $\mathrm{mm}$ sphere probe tips) were used: a metallic probe tip and a polytetrafluorethylene (PTFE) probe tip. A PI bar $(0.5 \mathrm{~mm} \times 5 \mathrm{~mm} \times 25 \mathrm{~mm})$ was used as the support of the

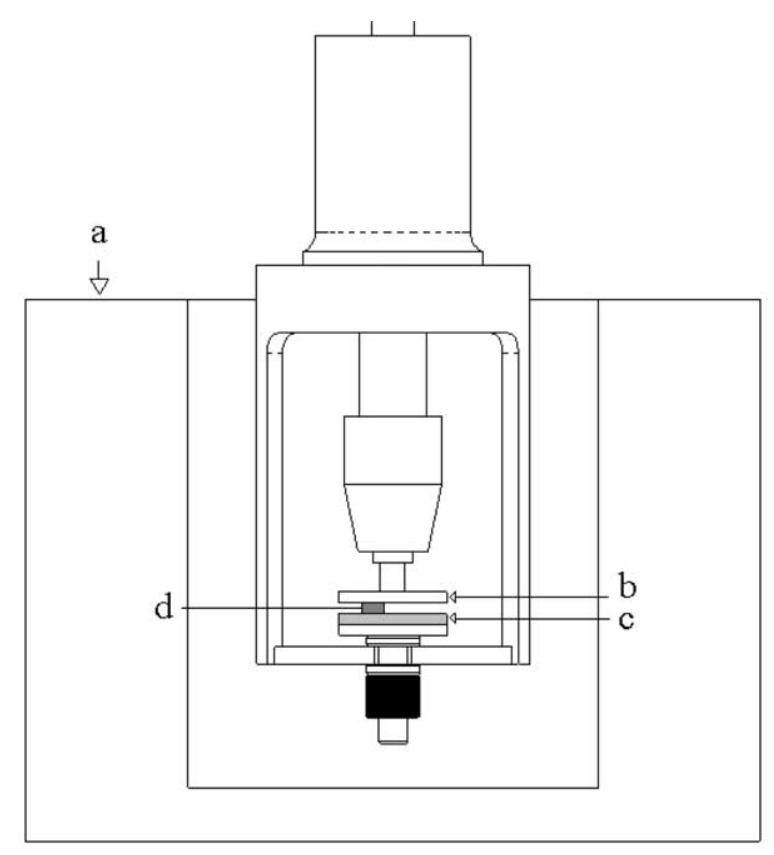

Fig. 1. Scheme of the arrangement used with the compression accessory for measuring the melting point of indium: $a=$ furnace; $\mathrm{b}=$ metallic plate; $\mathrm{c}=\mathrm{PI}$ film, $\mathrm{d}=$ indium sample. The furnace is shown is this figure in order to elucidate the arrangement of the assembly. standard. As for the real sample, it was located over the three-point bending platform edges.

In order to measure $T_{\mathrm{m}}$ at different points along the PI bar length, an adapted device was designed in our laboratory that permits changing the position of the probe tip, allowing performance of penetration experiments along the length of the sample. This device could be fixed to the probe shaft of the DMA apparatus (Fig. 2(b)). The experiments were performed either with a metallic probe tip or a ceramic probe tip (with similar dimensions to the DMA probe tips). The longest platform (length $=20 \mathrm{~mm}$ ) was used in this case. Experiments at several $\beta$ for two positions (centre and $9 \mathrm{~mm}$ away from the centre) were conducted.

\subsection{3. (iii) Extension}

The effect of $\beta\left(1 \leq \beta \leq 8^{\circ} \mathrm{C} \min ^{-1}\right.$ in $T_{\mathrm{m}}$ was investigated with the arrangement described in Fig. 3 for a fixed position and for two Helium flow rates $(v)\left(0 \mathrm{~cm}^{3} \mathrm{~min}^{-1}\right.$ and $28 \mathrm{~cm}^{3} \mathrm{~min}^{-1}$ ). A rectangular thin PI film was fixed to the upper clamp, acting as a probe tip, and a PI support constituted by a rectangular film glued (with an epoxy adhesive) to a square film for placing the indium sample was fixed to the lower clamp. PI films and supports with the same areas but with different lengths were used in order to locate the indium sample at different positions between the clamps. The position of the indium sample between clamps was varied in several ways:

(a) the distance from the indium sample to the upper clamp $\left(h_{\mathrm{u}}\right)$ was fixed and the distance from the sample to the lower clamp $\left(h_{1}\right)$ varied;

(b) $h_{1}$ was fixed and $h_{\mathrm{u}}$ varied;

(c) both distances varied in such a way that the sample is always at the centre of the distance between clamps and $h_{\mathrm{t}}$ varied $\left(h_{\mathrm{u}}=h_{1}\right)$.

The influence of the purge gas in $T_{\mathrm{m}}$ was also investigated for this accessory. Flow rates from 10 to $60 \mathrm{~cm}^{3}$ $\min ^{-1}$ were used.

\subsection{Dynamic experiments}

Some dynamic experiments in the extension mode were also performed. Rectangular polymethyl methacrylate (PMMA) samples (supplied by Goodfellow, catalogue number ME303010) with constant dimensions $(10.2 \mathrm{~mm} \times 3.57 \mathrm{~mm} \times 1 \mathrm{~mm})$ were analysed at different $\beta\left(2,4\right.$ and $\left.8^{\circ} \mathrm{C} / \mathrm{min}\right)$. In this case a static stress of 0.48 $\mathrm{MPa}$ and a dynamic stress of $0.4 \mathrm{MPa}$ at a frequency $(f)$ of $1 \mathrm{~Hz}$ were applied. Both storage modulus $\left(E^{\prime}\right)$ and loss modulus $\left(E^{\prime \prime}\right)$ were recorded as a function of temperature. 

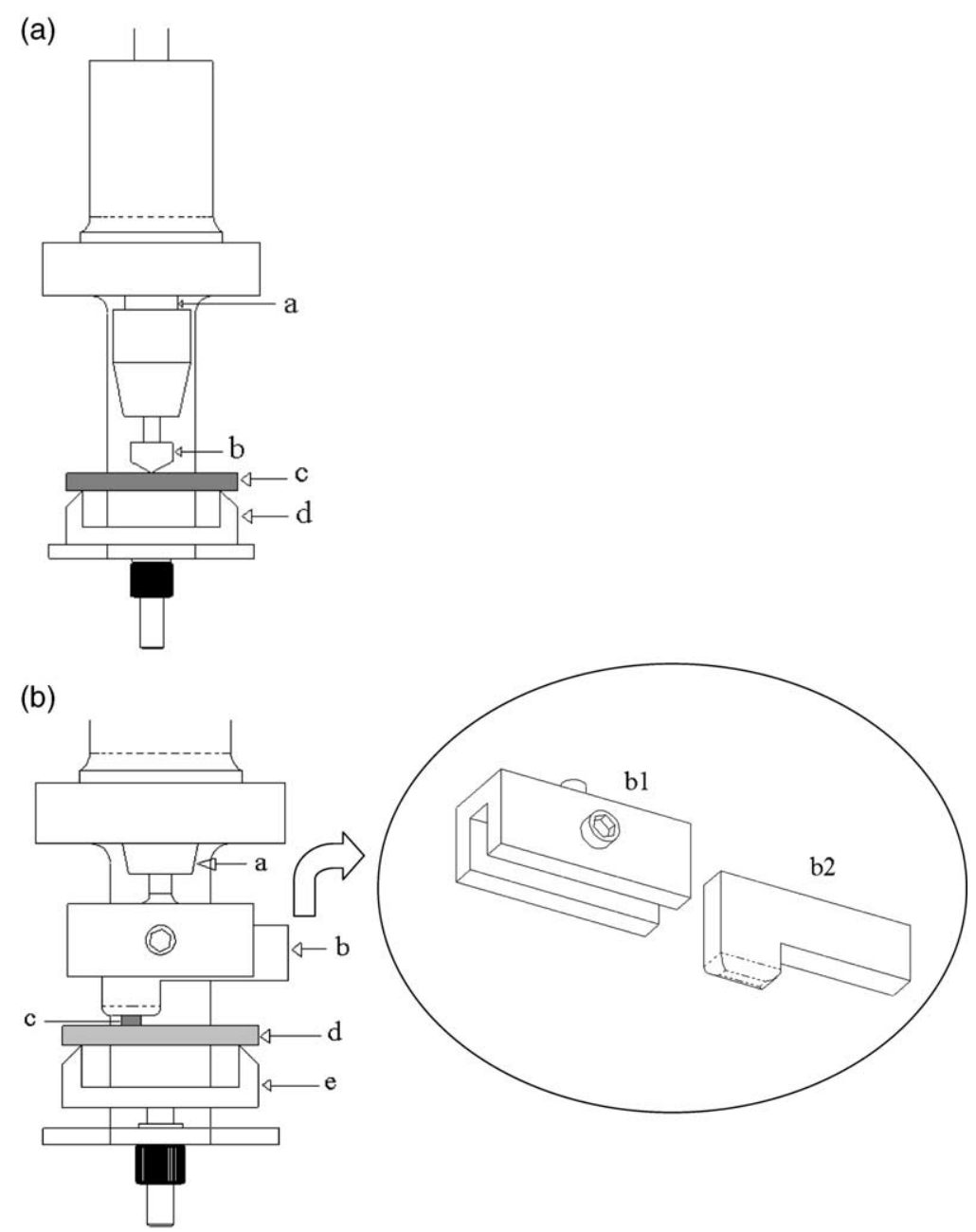

Fig. 2. Schemes of the arrangements used with the three-point bending accessory for measuring the melting point of indium ( $\left.T_{\mathrm{m}}\right)$. (a) Arrangement used for analysing the effect of the heating rate in $T_{\mathrm{m}}$ : a=probe shaft; $\mathrm{b}=$ probe tip; c=PI bar; $\mathrm{d}=\mathrm{bending}$ platform. (b) Arrangement used for analysing the effect of the distance between indium and the centre of the platform $\left(d_{\mathrm{f}}\right)$ in $T_{\mathrm{m}}$ : a=probe shaft; $b=$ adapted device; $c=$ indium sample; $d=P I$ bar; $e=$ bending platform. The adapted device is seen in more detail on the right side of the picture: it has a probe tip support (b1) with a screw for fixing the probe tip (b2) in the desired position along the groove of b1.

\section{Results and discussion}

The furnaces of DMA and TMA analysers have considerable dimensions in order to accommodate the distinct accessories and the sample, so one should expect important thermal gradients in the environment of the sample. In the case of the DMA used in this work the furnace is a platinum wire wound ceramic furnace with diameter $28 \mathrm{~mm}$ and height $45 \mathrm{~mm}$. The results are analysed separately for the three distinct modes of measurement.

\subsection{Compression}

In Fig. 4 is shown the effect of the distance from the centre of the plate $\left(d_{\mathrm{c}}\right)$ in the measured melting point of indium $\left(T_{\mathrm{m}}\right)$ for the compression accessory. A heating rate of $\beta=4^{\circ} \mathrm{C} \min ^{-1}$ was used in all experiments

In a first set of experiments the standard was located directly between the metallic plates (squares). As $d_{\mathrm{c}}$ increases a slight decrease in $T_{\mathrm{m}}\left(\sim 2^{\circ} \mathrm{C}\right)$ is observed. The heat transfer is made essentially by radiation and by convection promoted by the gas flux. The observed results can be explained if heat is irradiated from the periphery 


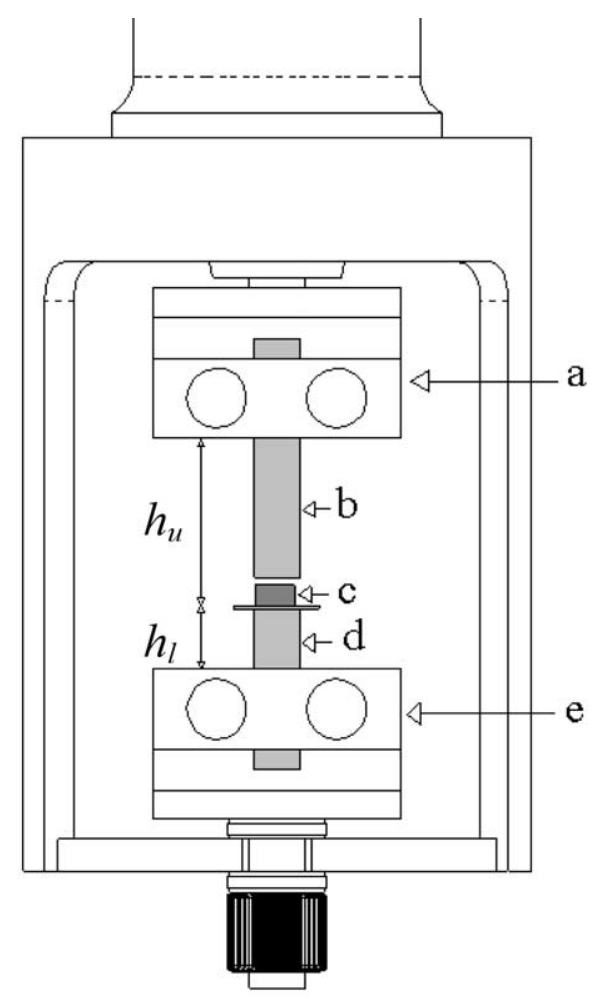

Fig. 3. Scheme of the arrangement used with the extension accessory for measuring the melting point of indium: $a=$ upper clamp; $b=P I$ film acting as a probe tip, $c=$ indium sample, $d=P I$ support for the standard; $\mathrm{e}=$ lower clamp. The distance between clamps $\left(h_{\mathrm{t}}\right)$ is given by $h_{\mathrm{t}}=h_{1}+h_{\mathrm{u}}$.

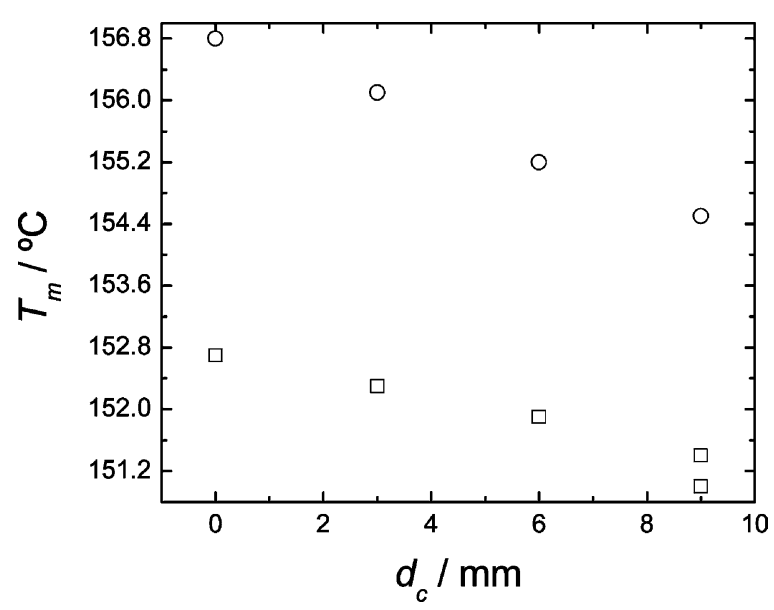

Fig. 4. Measured melting point of indium $\left(T_{\mathrm{m}}\right)$ against distance from the centre of the plate $\left(d_{\mathrm{c}}\right)$ for the compression accessory. $\square=$ experiments performed with the indium sample directly between the plates; for the distance of $9 \mathrm{~mm}$ the point at highest temperature was obtained with the indium sample nearest the thermocouple than the other point obtained at the same distance. $\bigcirc=$ experiments performed with the indium sample over a PI film between the plates. to the centre of the furnace. Hence, as we get closer to the centre of the plate, the surfaces are getting colder and it is necessary to attain a higher temperature for the indium to melt.

If we put a PI film between the indium and the plate (to try to simulate the presence of a sample as in a true experiment) the observed tendency is the same although the values of $T_{\mathrm{m}}$ are higher because the film is a bad heat conductor (circles in Fig. 4). It is also seen that there is a radial symmetry in the variation of $T_{\mathrm{m}}$ with $d_{\mathrm{c}}$. For the same $d_{\mathrm{c}}, T_{\mathrm{m}}$ is approximately the same, independently of the position of indium relative to the thermocouple.

\subsection{Three-point bending}

The effect of the distance from the centre of the PI bar $\left(d_{\mathrm{f}}\right)$ in $T_{\mathrm{m}}$, for the three-point bending mode, can be seen in Fig. 5. The usual probe tip of the three-point bending mode (Fig. 2(a)) was substituted by the accessory shown in Fig. 2(b) that can accommodate different probe tips and allows variation of $d_{\mathrm{f}}$ along the PI bar.

When a metallic probe tip is used $T_{\mathrm{m}}$ does not change with $d_{\mathrm{f}}$ (squares in Fig. 5). If we use a ceramic probe tip, instead of the metallic one, an important decrease of $T_{\mathrm{m}}$ with $d_{\mathrm{f}}$ (circles) is observed. The difference between measuring $T_{\mathrm{m}}$ in the centre of the platform or near the edges is about $10^{\circ} \mathrm{C}$. Moreover, the values measured with this probe tip are much lower $\left(\sim 20^{\circ} \mathrm{C}\right)$ than the ones measured with the metallic probe tip. This means that the point which is in contact with the metallic probe is around $20^{\circ} \mathrm{C}$ cooler than the reading of the thermocouple. The explanation for these results is that the metallic probe tip, the probe shaft and the accessory used to change the position of the probe tip remove heat from

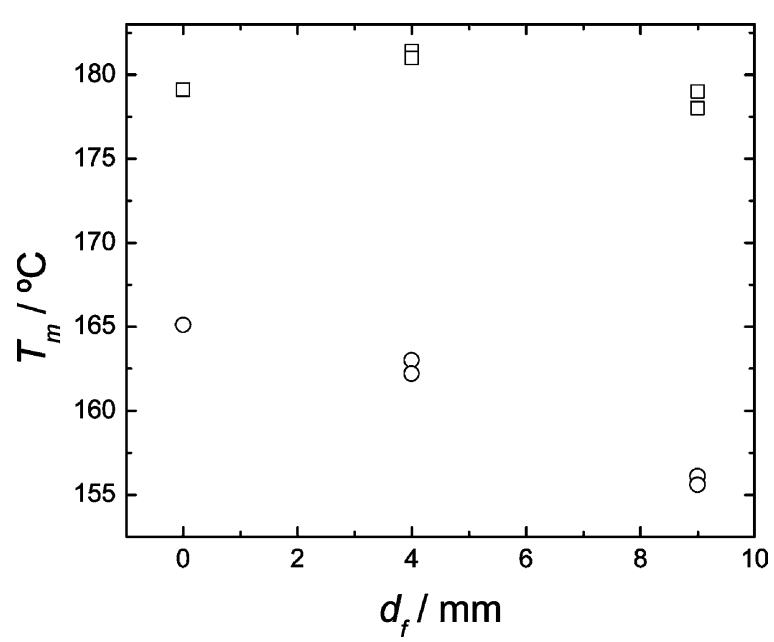

Fig. 5. Measured melting point of indium $\left(T_{\mathrm{m}}\right)$ against the distance from the centre of the PI bar $\left(d_{\mathrm{f}}\right)$ using the arrangement shown in Fig. 2(b). Two distinct probe tips were used: $\square=$ metallic probe tip, $\mathrm{O}=$ ceramic probe tip. 
the sample surroundings and from the sample itself. In fact, the purge gas (that enters at lower temperatures than $T_{\mathrm{m}}$ ) flows through the furnace from the top, passing primarily to the metallic probe tip. This explains the strong decrease of the $T_{\mathrm{m}}$ values when we put a bad heat conductor, like a ceramic probe tip, between the sample and the rest of the accessory. When there is a poor heat transmission between the sample and the upper accessory, and supposing that heat is irradiated from the periphery to the centre of the furnace, we should expect the observed decrease of $T_{\mathrm{m}}$ as $d_{\mathrm{f}}$ increases. In this case the thermal gradients involved are much higher than the ones detected with the compression mode, probably because the indium sample is placed above a PI bar (bad heat conductor) in order to simulate the presence of a sample in a true DMA experiment. On the contrary, in the compression mode we have two metallic plates in contact with the sample and very close with each other so it was not surprising to find smaller temperature gradients in the inner surfaces of the plates.

\subsection{Extension}

In the case of the extension mode it is more difficult to analyse the effect of the indium sample position on $T_{\mathrm{m}}$ because we have several factors: besides the distance between clamps $\left(h_{\mathrm{t}}\right)$, the probe can be at different distances from the upper clamp $\left(h_{\mathrm{u}}\right)$ and from the lower clamp $\left(h_{1}\right)$. The three effects were analysed independently. The observed melting temperatures of Indium are shown in Fig. 6 as a function of the total distance between clamps.

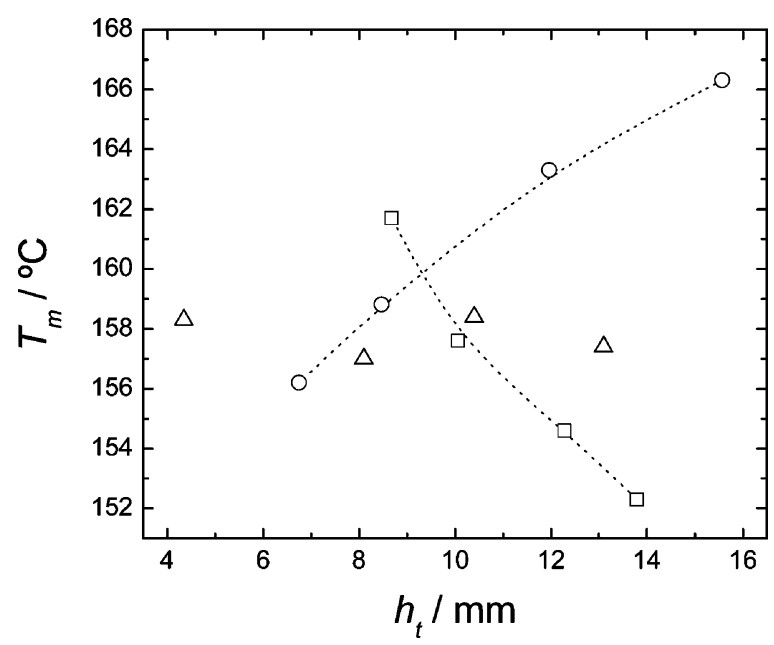

Fig. 6. Measured melting point of Indium $\left(T_{\mathrm{m}}\right)$ against the distance between clamps $\left(h_{\mathrm{t}}\right)$ for the extension accessory. $\square=$ the distance between the Indium sample and the lower clamp was fixed $\left(h_{1}=4.2 \mathrm{~mm}\right) ; \bigcirc=$ the distance between the sample and the upper clamp $\left(h_{\mathrm{u}}\right)$ was fixed $\left(h_{\mathrm{u}}=4.8 \mathrm{~mm}\right) ; \triangle$ =both distances varied in such a way that the sample is always at the same distance from the two clamps $\left(h_{\mathrm{l}}=h_{\mathrm{u}}\right)$.
For a fixed $h_{1}$ it was found that $T_{\mathrm{m}}$ decreases when $h_{\mathrm{t}}$ increases (squares in Fig. 6). This decrease is about $10^{\circ} \mathrm{C}$ for $8<h_{\mathrm{t}}<14 \mathrm{~mm}$. On the other hand, if $h_{\mathrm{u}}$ is maintained constant an increase of $T_{\mathrm{m}}$ as $h_{\mathrm{t}}$ increases is detected: $T_{\mathrm{m}}$ increases $\sim 10^{\circ} \mathrm{C}$ for $6.8<h_{\mathrm{t}}<15.6 \mathrm{~mm}$ (circles in Fig. 6). Looking at this set of results it seems that the lower clamp conducts heat to the sample and the upper clamp removes heat from the sample. So, when $h_{\mathrm{u}}$ is fixed the heat removed to the sample by this clamp is approximately the same, and as $h_{\mathrm{t}}$ increases $h_{1}$ also increases and the conduction of heat between the sample and the lower clamp is getting less effective. On the other hand, when $h_{1}$ is fixed the heat conducted to the sample by this clamp is approximately constant, and the increase of $h_{\mathrm{t}}$ is followed by an increase of $h_{\mathrm{u}}$, so the removal of heat performed by the upper clamp is not so efficient.

It is clear from the results in Fig. 6 that in the extension mode important temperature gradients along the distance between clamps were detected. It is interesting to note that when both distances $\left(h_{1}\right.$ and $\left.h_{\mathrm{u}}\right)$ varied in such a way that the sample is always at the centre of the distance between clamps, i.e. $h_{\mathrm{l}}=h_{\mathrm{u}}, T_{\mathrm{m}}$ is approximately the same. Apparently the opposite effects of the two clamps are counterbalanced when the indium sample is at the centre of the clamps.

Still in the extension mode, the influence of the purge gas flow rate (v) was investigated (Fig. 7). In all experiments the sample was at the same position.

The increase of $v$ leads to an increasing $T_{\mathrm{m}}$ that can be explained because there is a gas flow circulating with a higher rate that promotes the decrease of temperature in the sample environment. This variation is about $20^{\circ} \mathrm{C}$ for $10<v<60 \mathrm{~cm}^{3} \mathrm{~min}^{-1}$, being thus an important effect in the temperature calibration of such equipments.

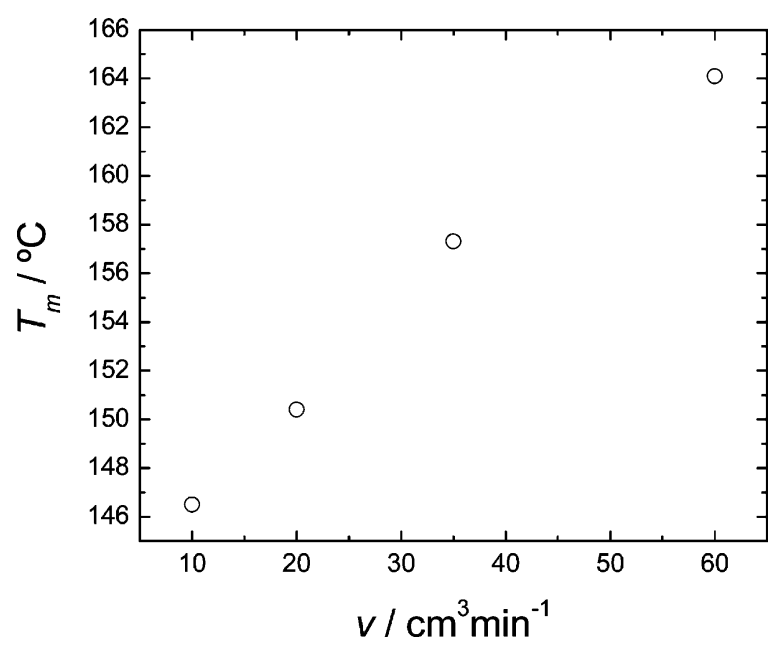

Fig. 7. Measured melting point of indium $\left(T_{\mathrm{m}}\right)$ for several flow rates of the purge gas $(v)$. These measurements were carried out with the extension accessory and the sample at a fixed position $\left(h_{1}=3.4 \mathrm{~mm}\right.$ and $\left.h_{\mathrm{t}}=10.3 \mathrm{~mm}\right)$. 


\subsection{Effect of the heating rate}

The effect of the heating rate $(\beta)$ of a run was analysed for the three modes. First consider the case of the compression accessory (Fig. 8), where the sample is located at a fixed position (centre of the plate) directly between the plates.

As expected, as $\beta$ increases, $T_{\mathrm{m}}$ increases due to the increase of the temperature lag $(\Delta T) . \Delta T$ increases with $\beta$ because as usual in thermal analysis equipments, the thermocouple is closer to the hot source than the sample. Assuming an ideal Newtonian behaviour $\Delta T$ is proportional to the heat flux, which increases with increasing $\beta$ and to the thermal resistance (essentially between the sample and the thermocouple) [5]. As reported before, $\beta$ has a strong effect on the measured values [11].

For the three-point bending mode the influence of $\beta$ in $T_{\mathrm{m}}$ is similar to that observed with the compression accessory, for identical reasons (Fig. 9). In this case the sample was located at the centre of the platform and two distinct probe tips were used.

With the metallic probe tip the measured values and the variation of $T_{\mathrm{m}}$ with $\beta$ are higher $\left(178<T_{\mathrm{m}}<190^{\circ} \mathrm{C}\right.$ for $1<\beta<8^{\circ} \mathrm{C} \min ^{-1}$ ) than with the PTFE probe tip $\left(155<T_{\mathrm{m}}<157.5^{\circ} \mathrm{C}\right.$ for the rate range used). The explanation given for the results of Fig. 6 may also explain this result if we consider that the metallic probe tip and the probe shaft remove heat from the sample and from the sample environment. This phenomenon is highly reduced when we put a bad heat conductor (PTFE) between the sample and the rest of the measuring system.

Some dynamic experiments in the three-point bending mode performed on a thermoset polymer at different heating rates and for a fixed frequency $(1 \mathrm{~Hz})$ confirm

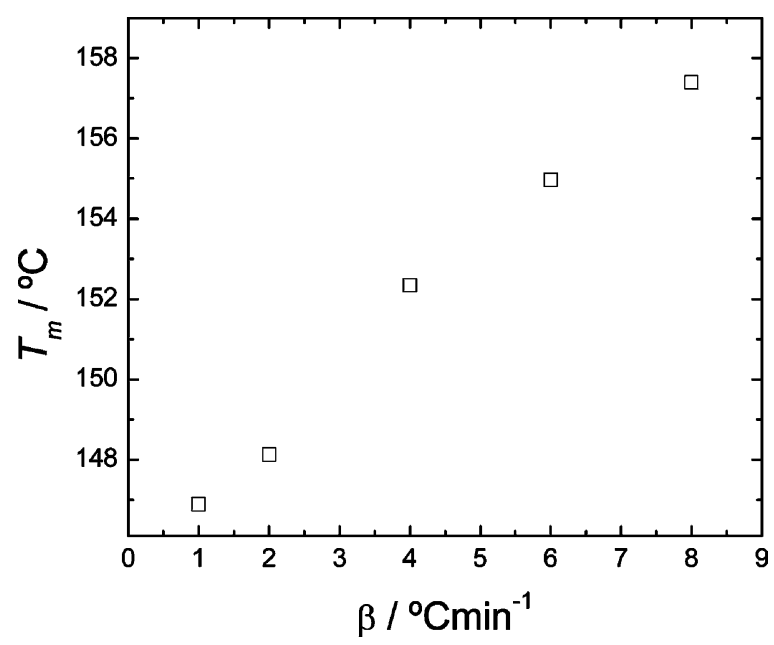

Fig. 8. Measured melting point of Indium $\left(T_{\mathrm{m}}\right)$ as a function of heating rate $(\beta)$ for the compression accessory and the indium sample located at the centre of the plate (directly between the plates).

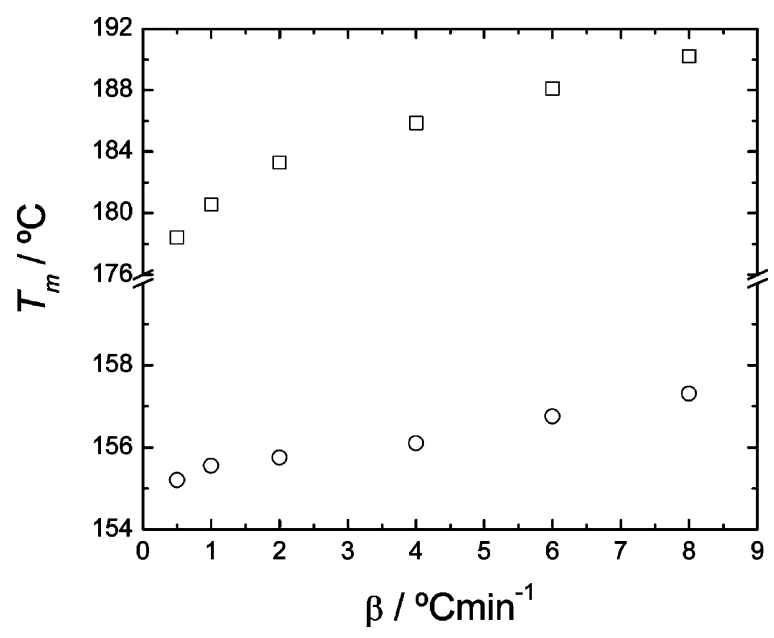

Fig. 9. Measured melting point of Indium $\left(T_{\mathrm{m}}\right)$ as a function of heating rate $(\beta)$ for the three point bending accessory and the indium sample located at the centre of the platform. Two distinct probe tips were used: $\square=$ metallic probe tip, $\bigcirc=$ PTFE probe tip.

the observed variation of $T_{\mathrm{m}}$ with $\beta$ [12]. In these results the $\alpha$-relaxation of the material, related to the dynamic glass transition, shifts to higher temperatures with increasing scanning rate.

For the extension mode an unexpected behaviour was detected for the variation of $T_{\mathrm{m}}$ with $\beta$ (Fig. 10). In fact, it is observed that $T_{\mathrm{m}}$ decreases as $\beta$ increases, i.e., as $\beta$ increases the temperature near the location of the standard is getting higher with respect to the temperature read by the sensor.

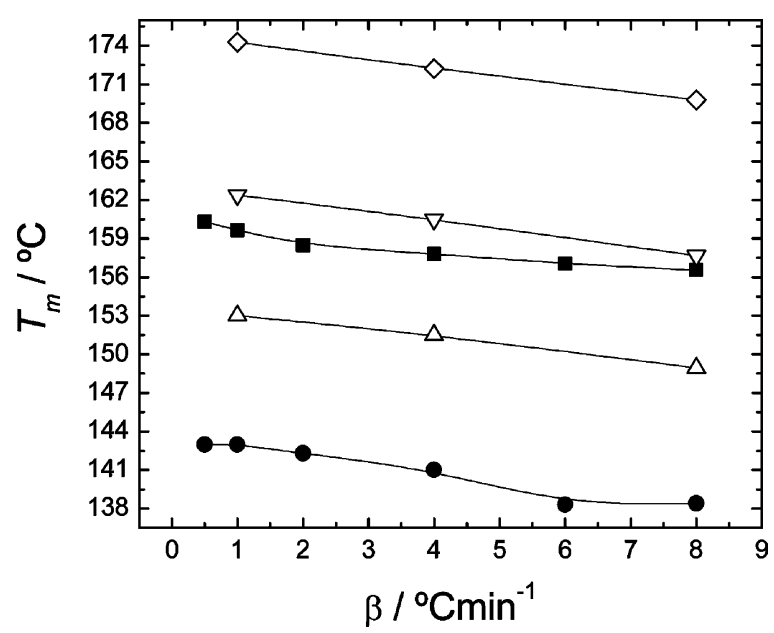

Fig. 10. Measured melting point of Indium $\left(T_{\mathrm{m}}\right)$ as a function of heating rate $(\beta)$ for the extension accessory. $\boldsymbol{\nabla}=h_{1}=4.2 \mathrm{~mm}$, $h_{\mathrm{t}}=10 \mathrm{~mm}, v=28 \mathrm{~cm}^{3} \mathrm{~min}^{-1} ; \boldsymbol{\Theta}=h_{1}=4.2 \mathrm{~mm}, h_{\mathrm{t}}=10 \mathrm{~mm}, v=0 \mathrm{~cm}^{3}$ $\min ^{-1} ; \Delta=h_{1}=3 \mathrm{~mm}, h_{\mathrm{t}}=14 \mathrm{~mm}, v=28 \mathrm{~cm}^{3} \min ^{-1} ; \nabla=h_{1}=8 \mathrm{~mm}$, $h_{\mathrm{t}}=14 \mathrm{~mm}, v=28 \mathrm{~cm}^{3} \mathrm{~min}^{-1} ; \diamond=h_{1}=12 \mathrm{~mm}, h_{\mathrm{t}}=14 \mathrm{~mm}, v=28$ $\mathrm{cm}^{3} \mathrm{~min}^{-1}$. 
This tendency was observed whenever the indium sample was located in different positions between clamps and for different flow rates (Fig. 10). In fact it can be seen that the tendency of decreasing $T_{\mathrm{m}}$ as $\beta$ increases is the same independently of the purge gas flow rate, although for a higher flow rate $T_{\mathrm{m}}$ is also higher (filled symbols). The explanation for this feature was given before. In the other set of experiments (open symbols) the flow rate and $h_{\mathrm{t}}$ were maintained constant and $h_{1}$ varied. The variation was identical but the $T_{\mathrm{m}}$ values were higher as $h_{1}$ increases because the sample is getting closer to the upper clamp (see explanation given for the results of Fig. 6). In our view, the only explanation for the unexpected tendency is the combination of the complex flow of the purge gas with the irradiation of heat from the furnace walls. Thus, a different trend may be found in other DMA/TMA equipment.

To confirm the decrease of $T_{\mathrm{m}}$ with increasing $\beta$ some dynamic experiments on PMMA samples with constant length at different $\beta$ were also performed in the region of the glass transition (Fig. 11) The temperature of maximum $E^{\prime \prime}$ of the $\alpha$-relaxation decreases with increasing $\beta$, which is in agreement with the tensile results obtained with the indium sample at different $\beta$.

The results corresponding to the open symbols of Fig. 10 give further information because for a fixed value of $\beta$ they simulate the measurement of the temperature in several points of a sample with fixed length. It is clearly shown that a temperature gradient exists along the sample length and for a distance between clamps of $14 \mathrm{~mm}$ this temperature gradient is about $20^{\circ} \mathrm{C}$. The results also suggest that the temperature of a sample in tensile conditions increases continuously as one goes from positions near the upper clamp down to the lower clamp.

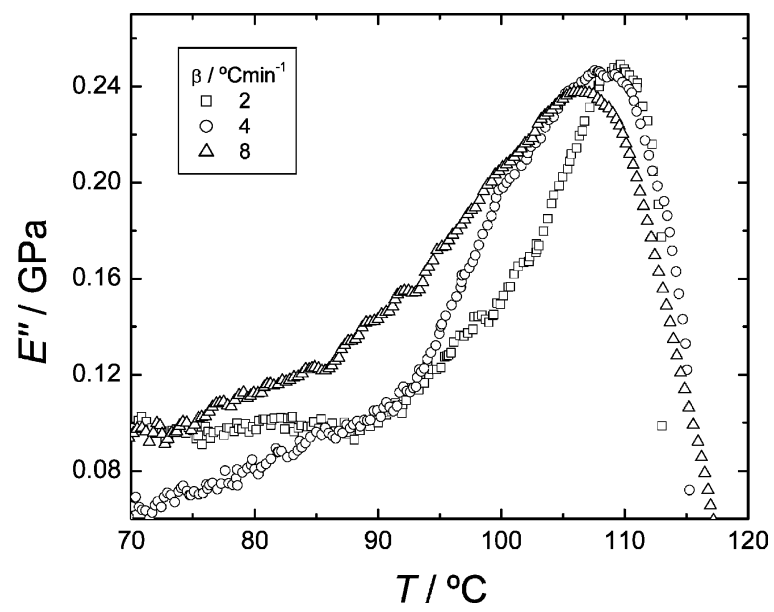

Fig. 11. Temperature dependence of the loss modulus $\left(E^{\prime \prime}\right)$ at the heating rates indicated in the plot $(f=1 \mathrm{~Hz})$. The experiments were conducted in the $\alpha$-relaxation region of PMMA with the extension mode.
It can also be seen in Fig. 10 that the difference between $T_{\mathrm{m}}$ values at two different positions is nearly independent of the value of $\beta$. This means that the change of the thermal gradients at the sample surface depends weakly on the scanning rate. Similar runs were performed for compression and three-point bending and once again considerable variations were not detected, especially in the former mode.

Another method for measuring thermal gradients along the sample length was previously reported, where high temperature differences were also found [13]. In this study experiments on fibre reinforced composites in flexural and torsional modes were carried out. A thermocouple was placed in the centre of the sample and the other was located in the area of the clamps at the centre of the sample in order to analyse the temperature gradient over the length of the test specimen. Experiments in two different DMA equipments revealed that within the free clamping region, a temperature gradient of up to $30^{\circ} \mathrm{C}$ may exist and, depending on the heating system of the furnace, a sample may be cooled or heated by the clamps.

It should be pointed out that, in practice, accurate dynamic mechanical measurements on non-isothermal experiments require that thermal equilibrium on the sample is achieved before starting the DMA experiments, as was emphasised in a recent work [14]. The constant values of $E^{\prime}$ and $\tan \delta$ displayed on the DMA unit before starting the run can be used as an indication that thermal equilibrium was attained. The authors found that the time required to attain this equilibrium (dependent on the heat transfer from the sample environment to the sample surface) is closely related to the sample position in the chamber and the distance between the sample and the thermocouple. For the extension mode the measured $E^{\prime}$ on heating was overestimated by two times and $\tan \delta$ shifts to higher values if they did not wait at least $15 \mathrm{~min}$ before starting the experiment. For the bending mode the time required to achieve thermal equilibrium is lower. It was also noted [14] that the increase of the sample length may result in temperature inhomogeneity along the sample length and a higher waiting time should be used.

\subsection{Simulation of the effect of the temperature gradients in the sample on dynamic mechanical measurements}

It was shown that mainly in the extension and threepoint bending modes of the studied apparatus considerable temperature gradients may exist at the surface of the samples. Such gradients can be amplified if one considers in addition the temperature profile inside the sample, caused by its own thermal resistance. As a step forward, it should be informative to analyse what should be the consequence of such thermal gradients on the final viscoelastic response of the sample, with respect to the 
ideal behaviour of existing a unique temperature in all the points of the sample. A simple model that assumes a linear change in temperature along the length of the sample (trying to simulate the behaviour of a sample found in tensile conditions) and no distribution of characteristic times for the response of the system at a given temperature will constitute a first approach to this problem. It is assumed that during the experiment the sample does not suffer chemical transformations (e.g. degradation or cure reactions) and does not melt. The temperature dependence of the single characteristic time is assumed to follow the Arrhenius equation.

Let the sample be divided along its length into $N$ parts, with equal lengths at zero stress (Fig. 12). If at zero dynamic stress the length of the sample is $l$, the length of each part, $l_{j}$ (where $j$ varies from 1 to $N$ ) is $l / N$. Under stress, the dynamic strain of the component $j$ is $\varepsilon_{j}^{*}=$ $\Delta l_{j}^{*} / l_{j}=N \Delta l_{j}^{*} / l$, where $\Delta l_{j}^{*}$ is the amplitude of the displacement of the component $j$ during the dynamic stress. The total strain is

$\varepsilon *=\sum_{j=1}^{N} \Delta l_{j}^{*} / l_{j}=\frac{\sum_{j=1}^{N} \Delta l_{j}^{*}}{l}=\frac{\sum_{j=1}^{N} \varepsilon_{j}^{*}}{N}$.

The dynamic mechanical compliance of the sample $D *=D^{\prime}-i D^{\prime \prime}=1 / E *$ is

$$
\begin{aligned}
& D *=\frac{\varepsilon^{*}}{\sigma *}=\frac{\sum_{j=1}^{N} \varepsilon_{j}^{*}}{N \sigma *}=\frac{1}{N} \sum_{j=1}^{N} D_{j}^{*}=\frac{1}{N} \sum_{j=1}^{N} D_{j}^{\prime} \\
& -i \frac{1}{N_{j=1}^{N} D_{j}{ }_{j},}
\end{aligned}
$$

where $D_{j}^{*}$ is the mechanical compliance of the component $j$, i.e., $\varepsilon_{j}^{*} / \sigma^{*}$. The loss factor is

$$
\tan \delta=\frac{D^{\prime \prime}}{D^{\prime}}=\frac{\sum_{j=1}^{N} D^{\prime \prime}{ }_{j}}{\sum_{j=1}^{N} D^{\prime}{ }_{j}} .
$$

If a relaxation process is characterised by a single characteristic time, $\tau$, the dynamic mechanical compliance $D *=D^{\prime}-i D^{\prime \prime}=1 / E *$, may be written, at a given temperature, as

$$
\begin{aligned}
D * & =D_{\mathrm{u}}+\frac{D_{\mathrm{r}}-D_{\mathrm{u}}}{1+i \omega \tau}=D_{\mathrm{u}}+\frac{D_{\mathrm{r}}-D_{\mathrm{u}}}{1+\omega^{2} \tau^{2}} \\
& -i \omega \tau \frac{D_{\mathrm{r}}-D_{\mathrm{u}}}{1+\omega^{2} \tau^{2}},
\end{aligned}
$$

where $i=(-1)^{1 / 2}, \omega$ is the angular frequency (linked to the frequency by $\omega=2 \pi f$ ) and $D_{\mathrm{u}}$ and $D_{\mathrm{r}}$ are the unrelaxed and relaxed mechanical compliances, respectively (i.e. at high and zero frequencies). Such an expression may be obtained from the analysis of a simple linear solid standard, where an elastic spring is linked in parallel with a second spring and a viscous element in series (see Fig. 12).

We will assume that $D_{\mathrm{u}}$ and $D_{\mathrm{r}}$ are temperature independent and that $\tau(T)=\tau_{0} \exp \left(E_{\mathrm{a}} / R T\right)$, where $\tau_{0}$ is a preexponential factor, $\mathrm{R}$ is the gas constant and $E_{\mathrm{a}}$ is the activation energy. Therefore, the differences in $D_{j}^{*}$ are due to the differences in $\tau$, because of the temperature gradient along the sample, which varies concomitantly with $j$, from $T-\Delta T / 2$ at $j=1$ to $T+\Delta T / 2$ at $j=N$ (the symmetric case, i.e. the decrease of temperature between $j=1$ and $j=N$, would lead to the same final results). Therefore, the difference in temperature between two adjacent components of the sample is $\Delta T /(N-1)$. It is then very easy, for a given temperature at the centre of the sample $T$ to compute the various $D^{\prime}{ }_{j}$ and $D^{\prime \prime}{ }_{j}$ with eq. (4). Eqs. (2) and (3) are used to calculate the measured $D^{\prime}(T)$, $D^{\prime \prime}(T)$ and $\tan \delta(T)$ of the sample, that can be compared with the values of the same variables if the temperature of the sample was homogeneous.

A simulation experience was carried out by assuming physically reasonable values for the variables: $D_{\mathrm{u}}=10^{-9}$ $\mathrm{Pa}^{-1}, D_{\mathrm{r}}=10^{-7} \mathrm{~Pa}^{-1}, E_{\mathrm{a}}=100 \mathrm{~kJ} \mathrm{~mol}^{-1}, \tau_{0}=10^{-14} \mathrm{~s}$, and $f=1 \mathrm{~Hz}$ (i.e. $\omega=2 \pi \mathrm{s}^{-1}$ ). The temperature $T$ varied between 40 and $200^{\circ} \mathrm{C}$. Various temperature gradients were tested: 0 (homogeneous case), 5, 10, 15, 20 and $30^{\circ} \mathrm{C}$. The results obtained were tested for different numbers of samples' parts, $N$. It was found that, for the stud-

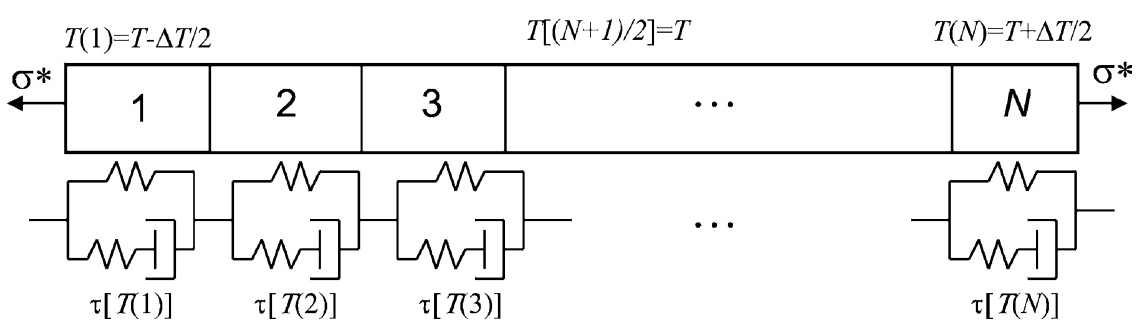

Fig. 12. Scheme of the model used in the simulations where it is assumed that there is a linear change in temperature along the length of the sample and no distribution of characteristic times for the response of the system at a given temperature. 
ied case, for $N$ higher than about 50, the viscoelastic parameters are almost independent of $N$; a value of $N=101$ was used in all simulations.

Figure 13(a) shows $D^{\prime}(T)$ and $D^{\prime \prime}(T)$ for the studied system, under the different temperature gradients. No strong changes in the results are found for $\Delta T \leq 10^{\circ} \mathrm{C}$ (the same was observed for $E^{\prime}$ and $E^{\prime \prime}$ Fig. 13(b), where $\left.E *=E^{\prime}+\mathrm{i} E^{\prime \prime}\right)$. A clear deviation from the homogeneous case (solid line) is just observed for $\Delta T>10^{\circ} \mathrm{C}$, i.e., when large temperature differences are found in the sample. This could be clearly seen on a linear scale in the peak maximum region of $E^{\prime \prime}$ or $D^{\prime \prime}$ (Figs 13(a) and 13(b)), as can be observed for $\tan \delta$ (Fig. 13(c)). Above $\Delta T$ $=10^{\circ} \mathrm{C}$ a consistent deviation is found in the $\tan \delta$ curves, leading progressively to shorter peaks, with peak temperatures that shift to smaller values, with increasing $\Delta T$.

It should be noted that the assumptions used in the analysis are far from realistic. In real polymeric systems a distribution of characteristic times exists in relaxation processes. Moreover the limit $D_{\mathrm{u}}$ and $D_{\mathrm{r}}$ values are also temperature dependent. The Arrhenius model is valid for thermally activated processes, but the WLF or VogelFulcher-Tamman-Hesse equations are usually used to describe $\tau(T)$ near and above $T_{\mathrm{g}}$. The regular change of temperature along the sample is a simplification of what would happen in a real system. However, the simple approach used may give a qualitative provision of how the existence of temperature gradients in a sample affects the measurements in DMA.

It was found in a thermoset system that, with increasing heating rate, the height of the $\tan \delta$ peak of the glass transition decreased consistently [12]. The simulations obtained indicate that such results are explained by the increase of temperature heterogeneity in the sample as the heating rate increases. This study also reported an increase of the same peaks' intensity as the cooling rate increased. Therefore, it seems that for the case of this particular equipment, the temperature gradients within the sample may be reduced as the cooling of the sample accelerates.

\section{Conclusions}

With this work it was shown that the thermal environment of a sample inside a DMA or TMA furnace may vary considerably depending on the mode used and this mode (compression, three-point bending, extension) should be taken into account in the calibration procedure. In the compression mode a small variation of the measured melting point was found as a function of the radial distance. However, for both extension and three-point bending modes quite important variations were measured. In the former case the bottom clamp was found to be warmer than the upper one and in the latter mode higher temperatures are found in the extremities of the (a)

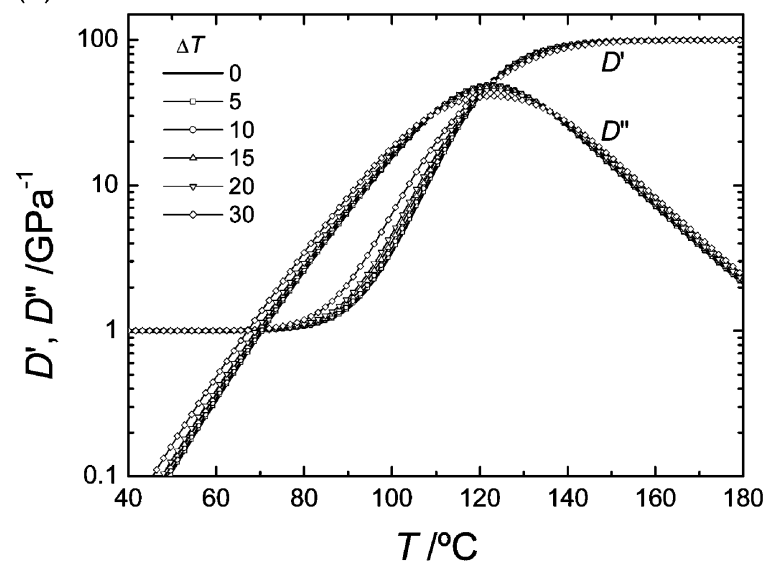

(b)

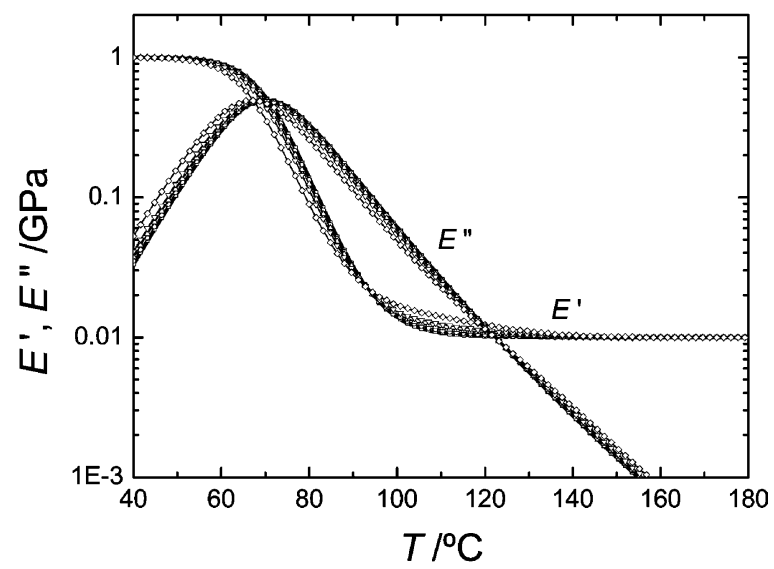

(c)

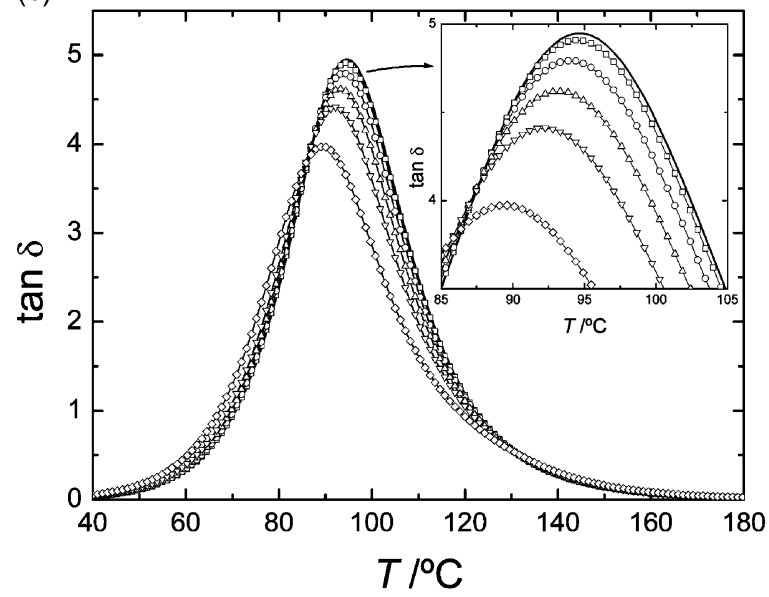

Fig. 13. (a) Storage compliance $\left(D^{\prime}\right)$, loss compliance $\left(D^{\prime \prime}\right)$, (b) storage modulus $\left(E^{\prime}\right)$, loss modulus $\left(E^{\prime \prime}\right)$ and (c) loss factor $(\tan \delta$ ) against temperature under the temperature gradients referred in the picture as predicted by the model. $D_{\mathrm{u}}=10^{-9} \mathrm{~Pa}^{-1}$, $D_{\mathrm{r}}=10^{-7} \mathrm{~Pa}^{-1}, E_{\mathrm{a}}=100 \mathrm{~kJ} \mathrm{~mol}^{-1}, \tau_{0}=10^{-14} \mathrm{~s}, f=1 \mathrm{~Hz}, N=101$ and $40<T<200^{\circ} \mathrm{C}$. 
samples (closer to the furnace wall). The knowledge of such temperature gradients and their dependence on the mode used may be useful when one attempts to correct the temperature read by the thermocouple, in order to estimate the sample temperature.

Some procedures for calibrating the temperature are now proposed. They attempt to improve the methods suggested in a previous work [12], taking into account the results reported in this study:

For the extension mode the melting point of the standard should be measured in the centre of the distance between clamps for a given experiment and the temperature correction will be calculated by subtracting this value from the theoretical one. The standard should be supported by a material with similar thermal conductivity to the sample to be studied.

In the method proposed for the three-point bending the melting point was already measured in the centre of the platform using a stiff material piece as sample holder. We suggest the use of a polymeric holder for the standard (a polymer whose glass transition temperature exceeds the melting point of the standard by at least $50^{\circ} \mathrm{C}$ ) instead of a metallic holder. In fact, the holder should have a thermal resistance similar to polymeric samples. A ceramic or polymeric probe tip should be used in order to reduce the heat removal performed by the metallic probe tip.

For the compression mode a temperature calibration performed with the plates used in the experiments and with the indium sample at the centre of the plate reflects better the environment felt by a sample than the conventional procedure. The standard could be placed between two plastic pieces, in order to probe the temperature inside a sample. This should provide the best approach to correct the temperature read by the DMA thermocouple in compression experiments.

The measured melting points are strongly dependent on the heating rate and on the purge gas flow rate for all modes, so the DMA calibration must be performed at the same heating rate and purge gas flow rate as the experiments.

From the simulation results (for a simple system) it can be concluded that, if significant thermal gradients exist along the sample length (above $10^{\circ} \mathrm{C}$ ), important changes may be found in $D *, E *$ and $\tan \delta$ measured by DMA.

\section{Acknowledgements}

NMA wishes to acknowledge Fundação para a Ciência e Tecnologia for the financial support trough the grant PRAXIS XXI/BD/20327/99.

\section{References}

[1] D. Campbell, J.R. White, Polymer Characterisation, Chapman and Hall, London, 1989.

[2] T. Leonard, G. Gooberman, An investigation into DMTA clamping problems, Meas. Sci. Technol 3 (1998) 275.

[3] P.L. Sullivan, D. Dykeman, Guidelines for performing storage modulus measurements using the TA instruments DMA three-point bend mode. I. Amplitude effects, Polym. Test 19 (2000) 155.

[4] M.A. Rodríguez-Pérez, O. Olmanza, J.L. del Valle, A. Gonzalez, J.A. Saja, Improvement of the measurement process used for the dynamic mechanical characterisation of polyolefin foams in compression, Polym. Test 20 (2001) 253.

[5] T. Hatakeyama, F.X. Quinn, Thermal Analysis-Fundamentals and Applications to Polymer Science, Wiley, 1994.

[6] R.J. Seyler, C.M. Earnest, Temperature calibration of thermomechanical analyzers: Part I-The development of a standard method, J. Test. Eval. 20 (1992) 430.

[7] R.J. Seyler, C.M. Earnest, Temperature calibration of thermomechanical analyzers: Part II-An interlaboratory test of the calibration procedure, J. Test. Eval. 20 (1992) 434.

[8] K.J. Kociba, Temperature calibration of TMAs using modulated temperature and Curie temperature reference standards, J. Therm. Anal. Cal. 60 (2000) 779.

[9] C. Lotti, S.V. Canevarolo, Temperature calibration of a dynamic-mechanical thermal analyser, Polym. Test 17 (1998) 523.

[10] R. Riesen, J.E.K. Schawe, Expansion and shrinkage of fibers-load and temperature modulated TMA measurements, temperature calibration of fiber attachments, J. Therm. Anal. Cal. 59 (2000) 337.

[11] J.F. Mano, Stress release in oriented HIPS as observed by dynamic mechanical analysis, Thermochim. Acta 332 (1999) 171.

[12] N.M. Alves, J.F. Mano, Temperature correction of dynamic mechanical and thermomechanical analysers during heating, cooling and isothermal experiments, Thermochim. Acta 346 (2000) 133.

[13] J. Wolfrum, G.W. Ehrenstein, M.A. Avondet, Dynamic mechanical thermo analysis of high performance composites-influences and problems, J. Compos. Mat. 34 (21) (2000) 1788 .

[14] I. Lacík, I. Krupa, M. Stach, A. Kucma, J. Jurciová, I. Chodák, Thermal lag and its practical consequence in the dynamic mechanical analysis of polymers, Polym. Test 19 (2000) 755 . 